\title{
Abdominal aortic aneurysm screening during transthoracic echocardiography in asymptomatic patients in Guilan province
}

\author{
Mohammad-Reza Aghajankhah Tamijani ${ }^{1}$, Negar Moghaddam², Hassan Moladoust*1 \\ Received: 5 Nov 2018 \\ Published: 27 Nov 2019
}

\begin{abstract}
Background: The prevalence of detected abdominal aortic aneurysm (AAA) during transthoracic echocardiography (TTE) has been different in previous studies based on the study population, and no data are available on AAA in the population of north of Iran. The aim of this study was to investigate the prevalence of AAA in individuals aged 50 and over in the north of Iran who were a candidate for TTE.

Methods: This cross sectional study was conducted on all individuals aged 50 and over who referred to our cardiovascular center for TTE evaluation from October 2016 to October 2017. The maximum diameter of the whole abdominal aorta was accepted as abdominal aortic size and a diameter $\geq 30 \mathrm{~mm}$ as AAA. All statistical analyses were conducted using SPSS Version 22.0. Also, Mann-Whitney and chi-squared tests were used to compare variables. A $\mathrm{p}<0.05$ was considered significant.

Results: In total, 1411 patients underwent TTE in this study and abdominal aorta was visualized in 1329 patients (93.9\%) successfully. The prevalence of AAA was $0.5 \%$ (7 patients, 95\% CI: 0.496-0.503) during the study period. Patients with AAA were significantly older $(\mathrm{p}=0.002)$, with a mean age of $74.4 \pm 7.7$ years, and $85.7 \%$ (6 patients) had hypertension, which was significantly higher $(\mathrm{p}=0.022)$ than patients without AAA.

Conclusion: This study showed that the AAA prevalence during standard TTE in the northern population of Iran aged 50 and over was $0.5 \%$, which was lower than a previous study in Tehran that found AAA on $3.8 \%$ of screened patients. Patients with AAA in this population were significantly older and more hypertensive.
\end{abstract}

Keywords: Abdominal aortic aneurysm, Aneurysm, Aorta, Echocardiography, Transthoracic

Conflicts of Interest: None declared

Funding: Vice Chancellor for research of Guilan University of Medical Sciences, Rasht, Iran

*This work has been published under CC BY-NC-SA 1.0 license.

Copyright $\odot$ Iran University of Medical Sciences

Cite this article as: Aghajankhah Tamijani MR, Moghaddam N, Moladoust $\mathrm{H}$. Abdominal aortic aneurysm screening during transthoracic echocardiography in asymptomatic patients in Guilan province. Med J Islam Repub Iran. 2019 (27 Nov);33:127. https://oi.org/10.47176/mjiri.33.127

\section{Introduction}

A pathologic local dilation of the abdominal aorta is called abdominal aortic aneurysm (AAA) (1). Many of AAAs are asymptomatic and develop with any noticeable symptoms over several years (2). AAAs detection is often an incidental finding when an imaging study is performed for another indication.

A ruptured abdominal aneurysm is an extremely lifethreatening condition that accounts for $2 \%$ of all deaths in men older than 65 years (3). Only $50 \%$ of these patients

\footnotetext{
Corresponding author: Dr Hassan Moladoust, hmoladoust@gums.ac.ir

1. Healthy Heart Research Center, Guilan University of Medical Sciences, Rasht, Iran

2. Heshmat Heart Hospital, Guilan University of Medical Sciences, Rasht, Iran
}

reach the hospital alive. The mortality rate before reaching the operating room is $70 \%$. Indeed, only up to $15 \%$ to $20 \%$ of these patients discharge from the hospital successfully (4-7). However, elective surgery of unruptured AAA decreases mortality and morbidity in these patients significantly (8). When the options of repairing thoracic endovascular aorta are limited and surgical morbidity is raised, elective surgery is suggested if the aortic diameter exceeds $6 \mathrm{~cm}$ or if there is a connective tissue disorder (9). Hence,

$\uparrow$ What is "already known" in this topic:

According to previous studies, abdominal aortic aneurysm (AAA) prevalence can be related to the screened population characteristics. Roshanali et al conducted a prospective study in Tehran and found AAA on $3.8 \%$ of screened patients.

$\rightarrow$ What this article adds:

It was found that the AAA prevalence in the northern population of Iran aged 50 and over was $0.5 \%$ of TTE screened patients. AAA were significantly older and more hypertensive. 
screening AAA in high-risk individuals is logical.

Transthoracic echocardiography (TTE) is one of the most commonly used imaging modalities for various purposes in every cardiology clinic. Many of these patients who are a candidate for TTE have the risk factors of AAA. TTE can detect structural heart disease and visualize the greater vessel and abdominal aorta. Screening AAAs during TTE has been evaluated in several singlecenter studies, whose results varied based on inclusion criteria and study population (10).

Screening AAAs during TTE has been evaluated in Roshanali et al study (11) at a general hospital in the Tehran. However, no data are available on the prevalence of this pathology in the northern population of Iran.

This study aimed to examine the prevalence of AAA in patients who were a candidate for TTE for different reasons at a referral cardiovascular center in Guilan province, north of Iran.

\section{Methods}

\section{Study population}

This cross sectional study was conducted on all consecutive patients aged 50 and over who were candidates for TTE with various indications and referred to a cardiovascular center in the north of Iran (Dr. Heshmat heart hospital in Rasht) from October 2016 to October 2017. Known or suspected aortic aneurysm or prior aortic aneurysm repair were the exclusion criteria.

Informed consent was obtained from all individuals to register their data. Age, gender, and body mass index (BMI) were recorded for all participants.

BMI was calculated as body weight $(\mathrm{kg})$ divided by the square of body height (m). A BMI of 18.5 to 24.9 was considered as normal range, a BMI of 25 to 29.9 as overweight, and BMI $\geq 30$ as obesity.

The patients' medical history, including history of hypertension, hyperlipidemia, diabetes mellitus, coronary arterial disease (CAD), cerebrovascular disease (CVD), and history of smoking, was reviewed.

Hypertension was considered as systolic blood pressure of $\geq 130 \mathrm{mmHg}$, diastolic blood pressure of $\geq 80 \mathrm{mmHg}$, and use of antihypertensive agents for previous hypertension (12). Diabetes mellitus was referred to the previous diagnosis of disease or use of insulin or antidiabetic agents in the patients' medical history (13). Diagnosis of hyperlipidemia was based on the previous detection of a condition or current usage of antihyperlipidemic agents. The diagnosis of CAD patients was based on a history of MI, coronary artery bypass surgery, or imaging or electrocardiographic findings (9). CVD was diagnosed based on previous ischemic stroke or transient ischemic attack (TIA) in patients' medical history.

The study protocol conformed to the ethical guidelines of the 1975 Declaration of Helsinki as reflected in a priori approval by the human research committee and was approved by the local ethics committee (IR.GUMS.REC.1394.277).

\section{Echocardiography}

All echocardiographic evaluations were performed us- ing an ACUSON SC2000 ultrasound system, equipped with a $1.25-4.5 \mathrm{MHz}$ transducer.

After a standard complete echocardiographic study, abdominal aorta was evaluated in patients with a supine position and a subcostal view for the suprarenal portion. The transducer was then moved as distal as possible to measure the infrarenal portion. The aortic segment image was first fixed in the systolic phase; then, its dimensions were measured from leading edge to leading edge using online electronic calibrators. Both longitudinal and transverse plane of the abdominal aorta were measured and the maximum diameter was accepted as abdominal aortic size.

AAA was defined as an increase in the size of the abdominal aorta to a size equal or more than $30 \mathrm{~mm}$ in diameter. Also, aortic dilation was defined as a diameter of $20 \mathrm{~mm}$ to less than $30 \mathrm{~mm}$. The diameter less than $20 \mathrm{~mm}$ indicated normal aortic size.

\section{Statistical analysis}

Statistical analyses were performed by SPSS software version 22.0 (SPSS Inc, Chicago, Illinois). KolmogorovSmirnov (KS) test was used to check normal distribution of continuous data. Also, a descriptive analysis was performed initially. Quantitative variables were reported as mean value \pm standard deviation (SD), and categorical variables were reported in numbers and percentages. Also, Mann-Whitney test was used to compare quantitative variables and chi-squared test was used for categorical variables. A p value less than 0.05 was considered statistically significant.

\section{Results}

In total, 1411 patients underwent TTE for various indications during the study period. Abdominal aorta was visualized in 1329 patients (93.9\%) successfully and included in the analysis. However, abdominal aorta could not be visualized adequately in 82 patients $(6.1 \%)$, and they were excluded from analysis. The most reasons for unsuccessful visualization of abdominal aorta were obesity and bowel gas.

The mean age of the study population was $61.5 \pm 7.5$ years (ranging from 50 to 90 years), of whom 642 patients (48.3\%, mean age of $62 \pm 7.2$ years) were male and 687 $(51.7 \%$, mean age $60.9 \pm 7.1$ years $)$ were female. The study demographic characteristics are illustrated in Table 1. The distribution of the abdominal aorta diameter is depicted in Figure 1.

Only 7 patients $(0.5 \%, 95 \%$ CI: $0.496-0.503)$ with AAA were found during the study period. The mean abdominal aorta diameter was $18.0 \pm 2.9 \mathrm{~mm}$ in all participants, and it was $31.7 \pm 2.8 \mathrm{~mm}$ in AAA group. The maximum measured diameter for abdominal aorta was $38.0 \mathrm{~mm}$. Also, 251 patients $(18.9 \%)$ had a dilated abdominal aorta diameter, with a mean diameter of $22.5 \pm 2.6 \mathrm{~mm}$.

Compared to the patients without AAA, those with AAA were significantly older, with a mean age of $74.4 \pm 7.7$ years $(p=0.002)$. Moreover, $85.7 \%$ of patients with AAA had hypertension, which was significantly higher $(\mathrm{p}=0.022)$ compared to patients without AAA.

Four patients $(57.1 \%)$ with AAA were male and 3 


\begin{tabular}{|c|c|c|c|c|c|}
\hline \multirow[b]{2}{*}{ Variables } & \multirow{2}{*}{$\begin{array}{l}\text { Total Patients } \\
\qquad(n=1329)\end{array}$} & \multicolumn{2}{|c|}{$\begin{array}{l}\text { AA without Aneurysm } \\
\qquad(\mathrm{n}=1322)\end{array}$} & \multirow{2}{*}{$\begin{array}{l}\text { Aneurysmal AA } \\
\qquad(\mathrm{n}=7)\end{array}$} & \multirow[t]{2}{*}{$\mathrm{p}^{*}$} \\
\hline & & $\begin{array}{c}\text { Normal AA } \\
(n=1071)\end{array}$ & $\begin{array}{c}\text { Dilated AA } \\
(n=251)\end{array}$ & & \\
\hline Age, mean (SD) & $61.5(7.5)$ & $61.18(7.3)$ & $62.52(8)$ & $74.4(7)$ & 0.002 \\
\hline Gender, n $(\%)$ & & & & & 0.639 \\
\hline Male & $642(48.3)$ & $513(47.9)$ & $125(49.8)$ & $4(57.1)$ & \\
\hline Female & $687(51.7)$ & $558(52.1)$ & $126(50.2)$ & $3(42.9)$ & - \\
\hline Diabetes Mellitus, n (\%) & $398(29.9)$ & $321(30)$ & 75 (29.9) & $2(28.6)$ & 0.936 \\
\hline Hypertension, n (\%) & $641(48.2)$ & $497(46.4)$ & $138(55.0)$ & $6(85.7)$ & 0.022 \\
\hline Hyperlipidemia, n (\%) & $523(39.4)$ & $425(39.7)$ & $97(38.6)$ & $1(14.3)$ & 0.173 \\
\hline Smoking, n (\%) & $367(27.6)$ & $281(26.2)$ & $84(33.5)$ & $2(28.6)$ & 0.955 \\
\hline CAD, n (\%) & $524(39.4)$ & $411(38.4)$ & $110(43.8)$ & $3(42.9)$ & 0.852 \\
\hline CVD, n (\%) & $30(2.3)$ & $17(1.6)$ & $13(5.2)$ & 0 & 0.687 \\
\hline
\end{tabular}

AA, Abdominal Aorta; SD, standard deviation; $n$, number; CAD, coronary arterial disease; CVD, cerebrovascular disease.

*At this column, we calculate $\mathrm{P}$ value for comparison between patients with and without AAA.

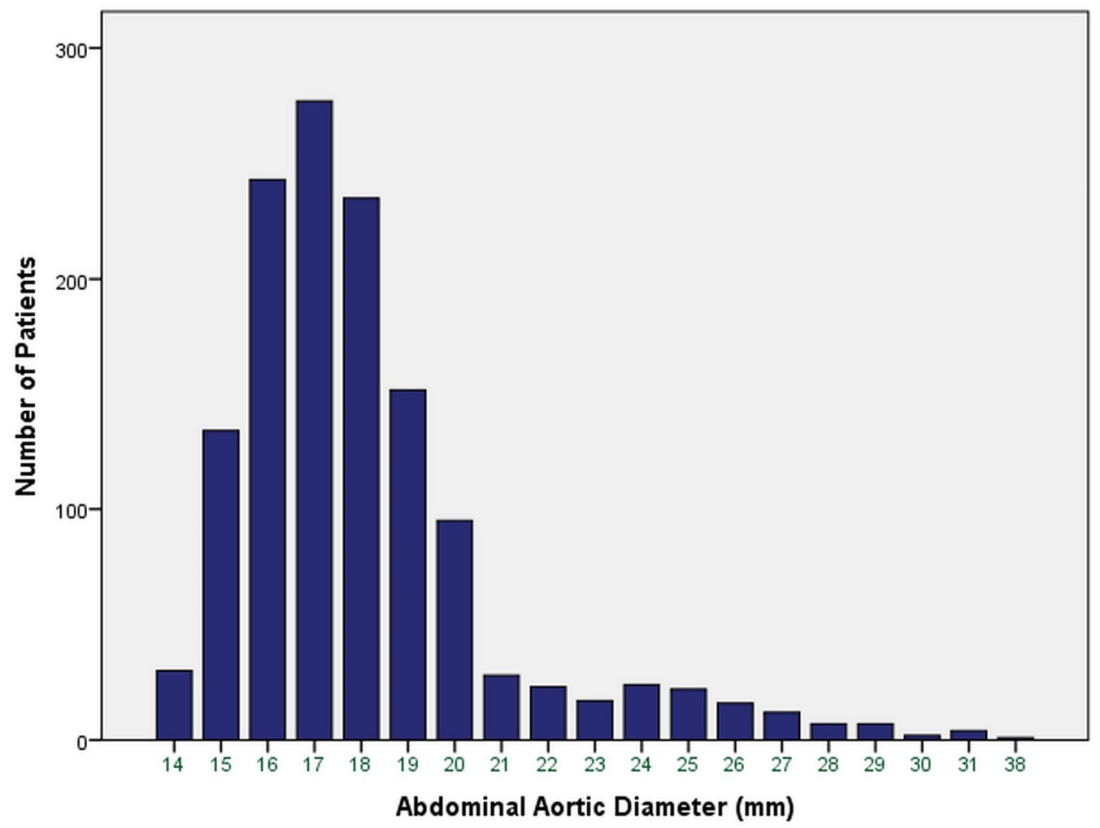

Fig. 1. Distribution of the abdominal aorta diameter

$(42.9 \%)$ were female. Distribution of gender did not differ in AAA group and in patients without AAA $(p=0.639)$. Based on the findings, prevalence of some characteristics in patients, including diabetes mellitus $(\mathrm{p}=0.936)$, hyperlipidemia $(\mathrm{p}=0.173)$, smoking $(\mathrm{p}=0.955)$, history of coronary arterial disease $(\mathrm{p}=0.852)$, and history of cerebrovascular disease $(\mathrm{p}=0.687)$, did not differ between participants with AAA and without AAA.

Distribution of BMI population is presented in Table 2. Most of the patients had a normal BMI (620 patients, $46.7 \%$ ) or were overweight (561 patients, $42.2 \%$ ). In
AAA group, 5 patients (71.4\%) had normal BMI. However, distribution of BMI did not differ between patients with and without AAA $(p=0.404)$.

\section{Discussion}

In the present study, the prevalence of asymptomatic AAA was evaluated in the population older than 50 years from north of Iran who were a candidate for TTE. Also, some demographic characteristics of these patients were assessed.

AAAs are the most common form of aortic aneurysms

Table 2. Distribution of BMI in the screened population

\begin{tabular}{|c|c|c|c|c|c|}
\hline \multirow[t]{2}{*}{ BMI } & \multirow{2}{*}{$\begin{array}{l}\text { Total patients } \\
\quad(n=1329)\end{array}$} & \multicolumn{2}{|c|}{$\begin{array}{l}\text { AA without Aneurysm } \\
(\mathrm{n}=1322)\end{array}$} & \multirow{2}{*}{$\begin{array}{l}\text { Aneurysmal AA } \\
\qquad(\mathrm{n}=7)\end{array}$} & \multirow[t]{2}{*}{$\mathrm{p}$} \\
\hline & & $\begin{array}{l}\text { Normal AA } \\
(\mathrm{n}=1071)\end{array}$ & $\begin{array}{c}\text { Dilated AA } \\
(n=251)\end{array}$ & & \\
\hline$<18.5$ & $49(3.7 \%)$ & $39(3.6 \%)$ & $10(4.0 \%)$ & 0 & 0.403 \\
\hline $18.5-24.9$ & $620(46.7 \%)$ & $523(48.8 \%)$ & $92(36.7 \%)$ & $5(71.4 \%)$ & \\
\hline $25-29.9$ & $561(42.2 \%)$ & $450(42.0 \%)$ & $110(43.8 \%)$ & $1(14.3 \%)$ & \\
\hline$\geq 30$ & $99(7.4 \%)$ & $59(5.5 \%)$ & $39(15.5 \%)$ & $1(14.3 \%)$ & \\
\hline
\end{tabular}

AA, Abdominal Aorta; $n$, number; BMI, body mass index 
and arise in infrarenal aorta (more than 80\%), the pararenal or visceral aorta (up to 10\%), and thoracoabdominal aorta. They are strongly and positively related to sex (fivefold in men), age, family history of AAA, cigarette smoking (fivefold), hypertension, emphysema, and hyperlipidemia (9).

Although a low prevalence of occult AAAs has been reported in previous literature $(2,14,15)$, it could be associated with serious life-threatening complications such as thromboembolic events due to a mural thrombi and ruptured abdominal aneurysm. Considering the high mortality rate of AAA rupture with or without surgery (3-7) and improved outcomes with elective repair before progression of complications (8), screening AAAs has been highly recommended. Indeed, aneurysm screening is associated with $50 \%$ reduction in rupture and mortality (16).

Some AAAs can be detected in physical examination as a pulsatile epigastric or periumbilical mass, particularly in thin patients with a large AAA. Nevertheless, the sensitivity of physical examination in detecting AAAs is only $30 \%-40 \%$, and many patients could be missed. Therefore, imaging has been turned as an important part of AAA screening (16).

When a pulsatile abdominal mass is present, ultrasonic imaging technique is recommended. Abdominal ultrasound can detect AAAs with high accuracy and is preferred because of its low cost, noninvasive, avoids radiation, and contrast agents. Since the accuracy of ultrasound in measuring AAA diameter is lesser than CT or MRI, ultrasound is just recommended for following up on small AAAs $(9,17)$. Based on U.S. Preventive Services Task Force in 2005 and the Society for Vascular Surgery, the AAA screening with ultrasound has been recommended for all men older than 65 years or for all men and women aged 55 and over with a family history of AAAs $(16,18)$. Therefore, in this study, it was aimed to evaluate the patients aged over 50 years.

Recently, many studies have evaluated the effectiveness of AAA screening during a standard TTE. The results of the literature vary based on different characteristics of studies. Some researchers limited the study population to a specific age range. For example, Spittell et al (19) and Seelig et al (20) only evaluated the patients over 50 years. Also, in Navas et al (21) cohort, only patients over 55 years were studied, but in the present study, the screened participants were limited to those aged 50 years and older. Some series studied selected populations such as patients with hypertension aged 50 and over (19), Asian population suffering from CAD (22), Korean population (23), patients admitted to cardiac care unit due to acute myocardial infarction (24), etc. However, others have investigated unselected participants $(11,25-29)$.

Different aortic segments have been imaged in different cohort studies. Some studies evaluated infrarenal abdominal aorta only (27-29) and others assessed suprarenal only (11). In routine echocardiography, ascending aorta, the root aorta, arch aorta, and suprarenal abdominal aorta are considered for dilatation and dissection, while infrarenal abdominal aorta assessment is not considered. As more than $80 \%$ of AAAs arise in the infrarenal aorta and the assessment is not time-consuming, in this study, it was aimed to investigate a whole abdominal aorta similar to some previous series (19-21). The diameter defined as AAA was also varied in the literature. However, most studies considered a diameter over $30 \mathrm{~mm}$ as AAA, similar to the present study.

Due to these discrepancies, the variable prevalence of detected AAA during TTE has been reported in different articles. Its prevalence ranges from $0.5 \%$ in Korean populations (23) to $19 \%$ in men older than 70 years in French populations (10). This significant difference may be due to the fact that the AAA prevalence could be associated with the characteristics of the screened population such as the risk factors of AAA formation, race, and gender. Therefore, the results were different from a previous study of Roshanali et al (11) in Iran. They conducted a prospective study in a general hospital of Tehran and found AAA on $3.8 \%$ of screened patients. However, the prevalence of detected AAA in this study was $0.5 \%$, similar to Korean populations in another cohort (23). This suggests that the prevalence of AAA in north of Iran differs from the rates found in Tehran. In Roshanali study, the mean age of the population was 40.7 years, and they evaluated only the suprarenal abdominal aortic segment and considered the diameter above $40 \mathrm{~mm}$ as abdominal aortic aneurysm. However, in the present study, the entire abdominal aorta was examined in an older population with a mean age of 61.5 years and also a smaller diameter (30 mm higher) was considered as the abdominal aortic aneurysm. In some previous studies, the maximum examination time for measuring the diameter of the suprarenal or infrarenal segment of the abdominal aorta was about 2 minutes (10, 11). In another cohort, viewing the whole abdominal aorta to screen AAA increased the total examination average time to 7.7 minutes (19). Although in this study the abdominal aorta examination time was not documented, the study of the whole abdominal aorta with TTE increased the time of echocardiography for only a few minutes. Moreover, in this study, abdominal aorta was evaluated in $93.9 \%$ of studied patients. This success rate was excellent, similar to the success rate of many previous studies (11, 19, 27-30). Furthermore, AAA screening as a routine part of TTE, did not impose any additional cost on the patients.

All these advantages suggest that AAA screening during TTE can be as a simple, quick, safe and cost-effective modality for screening this life-threatening condition.

However, this study could not show any relationship between AAA and such risk factors as CAD, CVD, male gender, smoking, hyperlipidemia, diabetes mellitus, and BMI. This result can be due to the low prevalence of AAA in this study. However, patients with AAA were older and hypertension was significantly more common in them. Simon et al (31) and Spittell et al (19) found that the incidence of AAA in hypertensive patients is significant $(3 \%$ and $11 \%$ for diastolic and systolic hypertensive patients \& $6.5 \%$, respectively).

This study had some limitations. First, the clinical history of the participants was collected retrospectively. Second, visualization of the abdominal aorta was inadequate in 82 patients $(6.1 \%)$ due to obesity and bowel gases, 
which could reduce the number of detected AAA and lead to bias. Moreover, a low prevalence of detected AAA in this study prevented form assessing a clinical risk model for AAA. Finally, in this study, no other imaging modalities were used to confirm the accuracy of TTE in measuring abdominal aorta diameter and screening an aneurysm. Moreover, patients were only selected from north of Iran. For generalizability of the results, conducting large, multicenter studies in other provinces of Iran and in different ethnic groups is highly recommended.

\section{Conclusion}

This study showed that the AAA prevalence during standard TTE in northern population of Iran aged 50 years and older was $0.5 \%$ and it was lower than a previous study in Tehran that found AAA on $3.8 \%$ of the screened patients. Patients with AAA in this population were significantly older and more hypertensive.

\section{Acknowledgements}

This study was financially supported in part by the Vice Chancellor for research of Guilan University of Medical Sciences, Rasht, Iran. We wish to thank the ethics committee (ethics code number: IR.GUMS.REC.1394.277, Thesis for degree of medical specialty) and all those who contributed to the completion of this study.

\section{Funding}

This work (Research project and Thesis for degree of medical specialty) was financially supported in part by Vice Chancellor for research of Guilan University of Medical Sciences, Rasht, Iran.

\section{Conflict of Interests}

The authors declare that they have no competing interests.

\section{References}

1.Sakalihasan N, Limet R, Defawe O. Abdominal aortic aneurysm. The Lancet. 2005;365(9470):1577-89.

2.Bickerstaff LK, Hollier LH, Van Peenen HJ, Melton LJ, Pairolero PC, Cherry KJ. Abdominal aortic aneurysms: the changing natural history. J Vasc Surg. 1984;1(1):6-12.

3.Collin J. Screening for abdominal aortic aneurysm. BJS Open. 1993;80(11):1363-4.

4.Kniemeyer $\mathrm{H}$, Kessler $\mathrm{T}$, Reber $\mathrm{P}$, Ris $\mathrm{H}$, Hakki H, Widmer M. Treatment of ruptured abdominal aortic aneurysm, a permanent challenge or a waste of resources? Prediction of outcome using a multi-organ-dysfunction score. Eur J Vasc Surg. 2000;19(2):190-6.

5.Wilmink TB, Quick CR, Hubbard CS, Day NE. The influence of screening on the incidence of ruptured abdominal aortic aneurysms. J Vasc Surg. 1999;30(2):203-8.

6.Adam DJ, Mohan IV, Stuart WP, Bain M, Bradbury AW. Community and hospital outcome from ruptured abdominal aortic aneurysm within the catchment area of a regional vascular surgical service. J Vasc Surg. 1999;30(5):922-8.

7.Bown M, Sutton A, Bell P, Sayers R. A meta-analysis of 50 years of ruptured abdominal aortic aneurysm repair. BJS Open. 2002;89(6):714-30.

8.Freiberg MS, Arnold AM, Newman AB, Edwards MS, Kraemer KL, Kuller LH. Abdominal aortic aneurysms, increasing infrarenal aortic diameter, and risk of total mortality and incident cardiovascular disease events: 10-year follow-up data from the Cardiovascular Health Study. Circulation. 2008;117(8):1010-7.
9.Zipes DP, Libby P, Bonow RO, Mann DL, Tomaselli GF. Braunwald's Heart Disease: A Textbook of Cardiovascular Medicine. eleventh ed: Elsevier Health Sciences; 2019.

10. Aboyans V, Kownator S, Lafitte M, Brochet E, Emmerich J, Tribouilloy C, et al. Screening abdominal aorta aneurysm during echocardiography: literature review and proposal for a French nationwide study. Arch Cardiovasc Dis. 2010;103(10):552-8.

11. Roshanali F, Mandegar MH, Yousefnia MA, Mohammadi A, Baharvand B. Abdominal aorta screening during transthoracic echocardiography. Echocardiography. 2007;24(7):685-8.

12. Whelton PK, Carey RM, Aronow WS, Casey DE, Collins KJ, Himmelfarb CD, et al. 2017 ACC/AHA/AAPA/ABC/ACPM/AGS/ $\mathrm{APhA} / \mathrm{ASH} / \mathrm{ASPC} / \mathrm{NMA} / \mathrm{PCNA}$ guideline for the prevention, detection, evaluation, and management of high blood pressure in adults: a report of the American College of Cardiology/American Heart Association Task Force on Clinical Practice Guidelines. J Am Coll Cardiol. 2018;71(19):127-248.

13. Association AD. Diagnosis and classification of diabetes mellitus. Diabetes care. 2014;37(Suppl 1):S81-S90.

14. Frame PS, Fryback DG, Patterson C. Screening for abdominal aortic aneurysm in men ages 60 to 80 years: a cost-effectiveness analysis. Ann Intern Med. 1993;119(5):411-6.

15. Wolk L, Pasdar H, McKeown JJ, Leibowitz H, Scott M. Computerized tomography in the diagnosis of abdominal aortic aneurysms. Surg Gynecol Obstet. 1981;153(2):229-32.

16. Chaikof EL, Brewster DC, Dalman RL, Makaroun MS, Illig KA, Sicard GA, et al. The care of patients with an abdominal aortic aneurysm: the Society for Vascular Surgery practice guidelines. J Vasc Surg. 2009;50(4):S2-S49.

17. Desjardins B, Dill KE, Flamm SD, Francois CJ, Gerhard-Herman MD, Kalva SP, et al. ACR Appropriateness Criteria ${ }^{\circledR}$ pulsatile abdominal mass, suspected abdominal aortic aneurysm. Int $\mathrm{J}$ Cardiovasc Imaging. 2013;29(1):177-83.

18. Moll F, Powell J, Fraedrich G, Verzini F, Haulon S, Waltham M, et al. Management of abdominal aortic aneurysms clinical practice guidelines of the European society for vascular surgery. Eur J Vasc Surg. 2011;41:S1-S58.

19. Spittell PC, Ehrsam JE, Anderson L, Seward JB. Screening for abdominal aortic aneurysm during transthoracic echocardiography in a hypertensive patient population. J Am Soc Echocardiogr. 1997;10(7):722-7.

20. Seelig M, Malouf Y, Klingler P, Oldenburg W, Atkinson E. Clinical utility of routine screening for abdominal aortic aneurysm during echocardiography. Vasa. 2000;29(4):265-8.

21. Navas EV, McCalla-Lewis A, Fernandez Jr BB, Pinski SL, Novaro GM, Asher CR. Abdominal aortic aneurysm screening during transthoracic echocardiography: cardiologist and vascular medicine specialist interpretation. World J Cardiol. 2012;4(2):31.

22. Lee SH, Chang SA, Jang SY, Lee SC, Song YB, Park SW, et al. Screening for abdominal aortic aneurysm during transthoracic echocardiography in patients with significant coronary artery disease. Yonsei Med J. 2015;56(1):38-44.

23. Oh SH, Chang SA, Jang SY, Park SJ, Choi JO, Lee SC, et al. Routine screening for abdominal aortic aneurysm during clinical transthoracic echocardiography in a Korean population. Echocardiography. 2010;27(10):1182-7.

24. Cueff C, Keenan NG, Krapf L, Steg PG, Cimadevilla C, Ducrocq G, et al. Screening for abdominal aortic aneurysm in coronary care unit patients with acute myocardial infarction using portable transthoracic echocardiography. Eur Heart J Cardiovasc Imaging. 2011;13(7):574-8.

25. Eisenberg MJ, Geraci SJ, Schiller NB. Screening for abdominal aortic aneurysms during transthoracic echocardiography. Am heart J. 1995;130(1):109-15.

26. Schwartz KV, Rashkow AM, Akella MS. Detection of abdominal aortic aneurysm during routine echocardiography. Echocardiography. 1996;13(1):71-3.

27. Jaussi A, Fontana P, Mueller X. Imaging of the abdominal aorta during examination of patients referred for transthoracic echocardiography. Schweiz Med Wochenschr Suppl. 1999;129(3):716.

28. Bekkers SC, Habets JH, Cheriex E, Palmans A, Pinto Y, Hofstra L, et al. Abdominal aortic aneurysm screening during transthoracic echocardiography in an unselected population. J Am Soc Echocardiogr. 2005;18(5):389-93.

29. Gentille-Lorente DI, Salvadó-Usach T. Screening for abdominal 
AAA Screening during TTE

aortic aneurysm by means of transthoracic echocardiography. Rev Esp Cardiol (Engl Ed). 2011;64(5):395-400.

30. Giaconi S, Lattanzi F, Orsini E, Prosperi R, Tartarini G. Feasibility and accuracy of a rapid evaluation of the abdominal aorta during routine transthoracic echocardiography. Ital Heart J. 2003;4(4):332-6.

31. Simon G, Nordgren D, Connelly S, Shultz PJ. Screening for abdominal aortic aneurysms in a hypertensive patient population. Arch Intern Med. 1996;156(18):2081-4 